\title{
Cytotoxic action of methylquercetins in human lung adenocarcinoma cells
}

\author{
KATRIN SAK ${ }^{1}$, HELEN LUST $^{1}$, MARJU KASE $^{2}$ and JANA JAAL ${ }^{1,2}$ \\ ${ }^{1}$ Clinic of Hematology and Oncology, Institute of Clinical Medicine, University of Tartu; \\ ${ }^{2}$ Department of Radiotherapy and Oncological Therapy, Tartu University Hospital, 51014 Tartu, Estonia
}

Received July 10, 2017; Accepted November 6, 2017

DOI: $10.3892 / 01.2017 .7466$

\begin{abstract}
Lung cancer is the malignant disorder associated with a high number of fatalities in women and men worldwide. Despite continuous improvements in diagnostic strategies and therapeutic modalities over the past decades, the prognosis and survival rate of patients suffering from lung cancer are still unsatisfactory and suggest the requirement for further molecular studies with different lung cancer models. In the present study, the anticancer action of two methylated metabolites of quercetin, isorhamnetin and tamarixetin, was assessed by studying their antiproliferative and apoptosis-inducing potential in human lung adenocarcinoma cell lines, A549 and HCC-44. Both methylquercetins decreased the viability of lung cancer cells at doses significantly lower than those effective for parent quercetin. The $\mathrm{IC}_{50}$ values measured for isorhamnetin were 26.6 and $15.9 \mu \mathrm{M}$ in A549 and HCC-44 cells, respectively. For tamarixetin, the $\mathrm{IC}_{50}$ values were 19.6 and $20.3 \mu \mathrm{M}$ in A549 and HCC-44 cells, respectively. These results were many-fold lower than the respective values for quercetin (72.2 and $107.6 \mu \mathrm{M}$ for A549 and HCC-44 cells, respectively). Based on the activation of caspase family members, both metabolites induced apoptotic cell death in the tested cell lines, predominantly via the extrinsic pathway in A549 cells and in both intrinsic and extrinsic pathways in HCC-44 cells. As A549 and HCC-44 lines were originally established from a male and female patient, current data may suggst some gender differences in the action of quercetin derivatives. Addition of a methyl group in the 3'- or 4'-position of the B-ring of quercetin significantly increased the anticancer activity of this flavonol towards lung adenocarcinoma cells, which demonstrated that these compounds may be considered as potential novel
\end{abstract}

Correspondence to: Dr Katrin Sak, Clinic of Hematology and Oncology, Institute of Clinical Medicine, University of Tartu, Ludvig Puusepa 8, 51014 Tartu, Estonia

E-mail:katrin.sak@ut.ee

Key words: non-small cell lung cancer, adenocarcinoma, flavonoids, methylated metabolites of flavonoids, quercetin, isorhamnetin, tamarixetin candidates for the development of future chemotherapeutics in the fight against lung cancer.

\section{Introduction}

Lung cancer has remained a global leading cause of cancer mortality in both men and women (1-3). In 2013, this malignant disorder constituted approximately 1.8 million new cancer cases and accounted for 1.6 million cancer deaths worldwide (2). Moreover, the number of lung cancer deaths is expected to grow up to 3 million for the year 2035 (3). Lung cancer is generally divided into two differently growing histological types, i.e., small-cell lung cancer and non-small cell lung cancer that accounts for the most of lung cancer cases (around 85\%) and includes adenocarcinoma, squamous cell carcinoma and large cell carcinoma $(1,3,4)$. Adenocarcinoma is the most common histological subtype that represents about $40 \%$ of all lung cancer cases $(1,4)$. Although men are more likely to be affected by this malignancy than women, with 1 in 18 men and 1 in 51 women diagnosed with lung cancer at some point in their lives, its incidence in women is globally increasing (3). Potential male-female differences have been demonstrated also in other aspects of lung cancer, including better prognosis, higher treatment responses and survival in women as compared to men (5-8). Nevertheless, the mean 5 -year survival rate of lung cancer is estimated to be less than $18 \%$, showing an urgent need for more effective treatment choices $(2,4)$. Recent bibliometric analysis revealed that international research level of lung cancer lags substantially behind the publication outputs for other malignancies. Despite the poor prognosis, high mortality rate and huge economic costs, in 2013 the research in lung cancer accounted only a small proportion, i.e., 5.6\% of all oncology research (2). Consequently, further in vitro and in vivo investigations using different lung cancer models are highly needed to develop novel treatment strategies and improve the survival rate of lung cancer patients.

In the recent years, several epidemiological studies have demonstrated that higher intake of fruits and vegetables can be beneficial for prevention of different types of human cancers, including lung tumors (9-13). As anticarcinogenic components of these plant products, polyphenolic flavonoids have been proposed with numerous experimental investigations to display various antitumoral activities (14-16). Indeed, 
these polyphenols can express antiproliferative, cytotoxic, proapoptotic, antiinvasive, antimetastatic, antiangiogenic and antiinflammatory properties in different cancer cell lines or animal models (17). However, it is well known that in the human body, flavonoids undergo an extensive metabolism and as a result of this conversion only different metabolic conjugates enter the circulatory system and can reach target malignant tissue $(18,19)$. Differently from parent flavonoids, current knowledge about the possible anticancer action of their metabolites is still rather limited making the prediction of bioactive behavior of flavonoids in the human body complicated.

One of the most important metabolic pathways that flavonoids undergo in the small intestine and liver is their methylation catalyzed by catechol-O-methyltransferase (COMT) (20). This phase II enzyme catalyzes the transfer of a methyl moiety from $\mathrm{S}$-adenosylmethionine donor substance to a catecholic substrate, such as flavonoids (21). As the methylated flavonoids formed in this way may potentially reveal substantially different biological properties than the parent compounds, we focus in this study on the anticancer effects and mechanisms of two methylated quercetin molecules, i.e., 3'-O-methylquercetin or isorhamnetin and 4'-O-methylquercetin or tamarixetin in human non-small cell lung cancer (adenocarcinoma) lines, A549 and HCC-44, and investigate their antiproliferative activities compared to the parent quercetin. In addition to studying the cell growth inhibitory effects of these methylated flavonoids by the MTT assay, their ability to trigger apoptotic pathways (i.e., intrinsic vs extrinsic routes) is also under examination by determining caspase- 9 and -8 activities. This investigation shows that flavonoid metabolites can be considered as leading compounds for further development of lung cancer chemotherapeutics possibly supplementing the available treatment arsenal in the future.

\section{Materials and methods}

Reagents. All flavonoids (genistein, daidzein, fisetin, quercetin, hesperetin, luteolin, chrysin, baicalein) and methylated derivatives of quercetin (isorhamnetin, tamarixetin) were purchased from Santa Cruz Biotechnology (Dallas, TX, USA). 3-(4,5-Dimethylthiazol-2-yl)-2,5-diphenyltetrazolium bromide (MTT) and L-glutamine were the products of Sigma-Aldrich (St. Louis, MO, USA). Dimethyl sulfoxide (DMSO) was from Mediatech, Inc. (Manassas, VA, USA). Phosphate-buffered saline (PBS) was obtained from Lonza (Verviers, Belgium).

Cell lines and culture conditions. A549 and HCC-44 human lung adenocarcinoma cell lines were obtained from the Leibniz Institute DSMZ-German Collection of Microorganisms and Cell Cultures (Leibniz-Institut DSMZ-Deutsche Sammlung von Mikroorganismen und Zellkulturen $\mathrm{GmbH}$, Braunschweig, Germany).

A549 and HCC-44 are cell lines derived from the adenocarcinoma of a 58-year-old Caucasian man (22) and a 54-year-old woman, respectively (23). To exclude clinically distinct disease entity-oncogene addicted lung cancer, these cell lines were further tested in our laboratory for epidermal growth factor receptor (EGFR) mutation and anaplastic lymphoma kinase (ALK) translocation. Both cell lines were wild type and did not have these genetic alterations (data not shown).

A549 cells were cultivated in Dulbecco's modified Eagle's medium (DMEM) (Life Technologies Corporation, Grand Island, NY, USA) supplemented with $10 \%$ of heat-inactivated fetal bovine serum (FBS; Invitrogen ${ }^{\mathrm{TM}}$, Auckland, NZ, USA). HCC-44 cells were cultivated in RPMI 1640 medium (Life Technologies Corporation) supplemented with $10 \%$ of heat-inactivated FBS. Cells were maintained in a $5 \% \mathrm{CO}_{2}$ incubator at $37^{\circ} \mathrm{C}$ with and passaged 1-2 times per week.

Measurement of cell viability by MTT assay. The cell growth inhibitory effects of flavonoids against human lung cancer cell lines were tested by the MTT colorimetric assay first described by Mosmann in 1983 (24). In detail, the cells were plated on to 96 -well $\mathrm{U}$ shaped bottom plates at concentration of $1 \times 10^{5}$ cells $/ \mathrm{ml}$ of medium, putting $100 \mu \mathrm{l}$ of suspension to each well. Cells were counted in Bürker counting chamber. As phenol red can interfere with the reading of absorbance (25), the cells were seeded in the phenol red-free RPMI-1640 medium (Mediatech, Inc.). After overnight culturing, cells were treated with varying doses of flavonoids (10 nM-500 $\mu \mathrm{M})$ for $48 \mathrm{~h}$ at $37^{\circ} \mathrm{C}$ and $5 \% \mathrm{CO}_{2}$. At the end of the incubation, $50 \mu \mathrm{l}$ of MTT solution in PBS was added to the wells with the final concentration of MTT of $5 \mathrm{mg} / \mathrm{ml}$. Plates were further incubated for $4 \mathrm{~h}$ followed by centrifugation at $1,000 \mathrm{rpm}$ for $10 \mathrm{~min}$ and removing of the supernatant. To dissolve the purple formazan crystals $150 \mu \mathrm{l}$ of DMSO was added and the plates were shaken for $30 \mathrm{~min}$. Absorbance was measured at $540 \mathrm{~nm}$ using a LED based microplate reader (Ledetect 96; Labexim Products, Lengau, Austria). To calculate the proportion of surviving cells, the following formula was used: (OD of drug-treated sample - OD of blank)/(OD of control - OD of blank) $\times 100 \%$, where OD of blank represents the absorbance reading of wells containing the buffer only (without cells) and OD of control represents the reading value of wells without any added test compounds. Dose-response curves were constructed to evaluate the half-maximal inhibitory concentrations $\left(\mathrm{IC}_{50}\right.$ values). All separate tests were carried out 2-3 times in different days, performing the experiments in triplicates.

Measurement of caspase activities. To study the cell death mechanisms triggered by the treatment of human lung cancer cell lines with methylated quercetin derivatives, the Caspase Colorimetric Protease Assay Sampler kit (Invitrogen Corporation, Frederick, MD, USA) was used according to the protocol provided by the manufacturer. As this kit uses para-nitroaniline-labeled synthetic peptides as substrates of different caspases, absorption of cleaved para-nitroaniline was spectrophotometrically quantified at $405 \mathrm{~nm}$.

Statistics. Data were treated using the GraphPad Prism statistical software (version 4.0; GraphPad Software, Inc., La Jolla, CA, USA). The Kolmogorov-Smirnov test for normality was applied and the one-way analysis of variance (ANOVA) was performed to determine whether the differences between means were statistically significant. P-value $<0.05$ were considered as statistically significant and all values were expressed as mean \pm standard deviation (SD). 


\section{Results}

Cytotoxicity profiles of flavonoids in human lung adenocarinoma cells A549 and HCC-44. Among the tested panel of flavonoids, isoflavones genistein and daidzein and flavanone hesperetin had no growth inhibitory effects on both lung cancer cell lines up to $100 \mu \mathrm{M}$ concentration. Flavonols fisetin and quercetin as well as flavones luteolin, chrysin and baicalein revealed low antiproliferative efficiency with half maximal inhibitory constants $\left(\mathrm{IC}_{50}\right)$ more than $100 \mu \mathrm{M}$, except quercetin in A549 cells with $\mathrm{IC}_{50}$ of $72.2 \mu \mathrm{M}$, and fisetin and chrysin in HCC-44 cells with $\mathrm{IC}_{50}$ values of 78.7 and $79.6 \mu \mathrm{M}$, respectively (Table I).

Growth inhibition of human lung adenocarcinoma cells A549 and HCC-44 by methylated metabolites of quercetin. Differently from the parent quercetin, its metabolites with one methyl group in the different positions of B-ring revealed much higher efficiency in inhibition of growth of lung cancer cells. In detail, the derivative containing a methyl moiety in the 3'-position, i.e., 3'-O-methylquercetin or isorhamnetin, displayed the inhibitory constants of 2.7- and 6.8-fold lower compared to the parent quercetin in $\mathrm{A} 549\left(\mathrm{IC}_{50}, 26.6 \mu \mathrm{M}\right)$ and HCC- 44 cells $\left(\mathrm{IC}_{50}, 15.9 \mu \mathrm{M}\right)$. The respective increases in cytotoxic potencies were 3.7- and 5.3-fold for 4'-O-methylquercetin or tamarixetin in $\mathrm{A} 549\left(\mathrm{IC}_{50}, 19.6 \mu \mathrm{M}\right)$ and $\mathrm{HCC}-44$ cells $\left(\mathrm{IC}_{50}, 20.3 \mu \mathrm{M}\right)$ (Table I). Chemical structures of these methylquercetins with the dose-response curves in both lung adenocarcinoma cell lines are presented in Fig. 1.

Effect of methylquercetins on activation of caspase family members. The effect of methylated quercetins on lung adenocarcinoma cell lines was further estimated by analysis of activity of caspase family members. In both A549 and HCC-44 cells, isorhamnetin increased caspase-3 activity by 2.5- and 3.5-fold, respectively, indicating induction of apoptosis. Similar results were measured for tamarixetin with the respective increases of 2.3- and 3.3-fold in A549 and HCC-44 cells. Furthermore, both derivatives elevated caspase- 8 activity in both cell lines pointing to the occurrence of apoptosis via extrinsic pathway. In HCC-44, but not in A549 cells, also the activity of caspase- 9 was increased demonstrating that in these cells the apoptosis is induced through both intrinsic and extrinsic routes (Fig. 2).

\section{Discussion}

A549 and HCC-44 cell lines are two non-small cell lung cancer lines derived from the adenocarcinoma of a 58-year-old Caucasian man (22) and a 54-year-old woman, respectively (23). The current work is the first study to describe the inhibitory effects of flavonoids on the viability of HCC-44 cells. As A549 line has been widely used as a model system to study human alveolar carcinoma, cytotoxic profile of flavonoids in these cells was previously characterized with the results very similar to those measured in our work. Indeed, the $\mathrm{IC}_{50}$ value of $72.2 \mu \mathrm{M}$ for quercetin is close to the inhibitory constants published by Loizzo et al (26), Tan et al (27-29), Robaszkiewicz et al (30), and Chan et al (31); and $\mathrm{IC}_{50}$ values more than $100 \mu \mathrm{M}$ were previously reported for fisetin (32), hesperetin (33-35),
Table I. Antiproliferative effects of flavonoids on human lung adenocarcinoma cell lines A549 and HCC-44.

\begin{tabular}{lcr}
\hline & \multicolumn{2}{c}{$\mathrm{IC}_{50}, \mu \mathrm{M}$} \\
\cline { 2 - 3 } Variable & $\mathrm{A} 549$ & $\mathrm{HCC}-44$ \\
\hline Isoflavones & & $>500$ \\
$\quad$ Genistein & $>500$ & $>500$ \\
Daidzein & & \\
Flavonols and their & & \\
methylated metabolites & $127.9 \pm 1.9$ & $78.7 \pm 2.0$ \\
Fisetin & $72.2 \pm 2.3$ & $107.6 \pm 2.2$ \\
Quercetin & $26.6 \pm 1.7$ & $15.9 \pm 1.7$ \\
3'-O-Methylquercetin & & $20.3 \pm 1.4$ \\
or isorhamnetin & $19.6 \pm 1.3$ & $>500$ \\
4'-O-Methylquercetin & & \\
$\quad$ or tamarixetin & & $123.0 \pm 1.6$ \\
Flavanones & & $79.6 \pm 1.6$ \\
Hesperetin & & \\
Flavones & & \\
Luteolin & & \\
Chrysin & & \\
Baicalein & $155.6 \pm 1.6$ & \\
\hline
\end{tabular}

luteolin (32,36), chrysin (35), baicalein (34), daidzein (37), and genistein (38). Based on our results presented in this article, cytotoxic activity profiles of flavonoids were rather similar for both A549 and HCC-44 lung cancer lines, despite the gender difference of initial origin of these cells.

However, the data clearly show that the growth inhibitory effects of tested flavonoids on lung cancer cells revealed only at very high micromolar doses that are physiologically unachievable. Indeed, the maximum serum concentrations of daidzein and genistein were measured to be less than $0.5 \mu \mathrm{M}$ following to consumption of $100 \mathrm{ml}$ of untreated soymilk (39). The baseline plasma concentration of quercetin was reported to be generally about $50-80 \mathrm{nM}$ reaching $0.63 \mu \mathrm{M}$ after supplementation with $80 \mathrm{mg}$ quercetin per day for one week or $1.5 \mu \mathrm{M}$ after supplementation with $>1 \mathrm{~g}$ quercetin per day for 4 weeks (40). Although bioavailability of flavonoids depends on food sources (for instance, quercetin is somewhat better bioavailable from onions than apples) and there is also high interindividual variability $(40,41)$, it is rather impossible to achieve plasma doses exceeding some micromolar level by oral ingestion of flavonoids-rich food items or dietary supplements.

Despite numerous experimental works performed with bioactivity of parent flavonoids, the knowledge about possible antiproliferative effects of their metabolites is still rather sparse today. One reason for this is the very limited commercial availability of metabolites for experimental testing. There are three major types of metabolic derivatives of flavonoids formed as a consequence of enzymatic conjugation with methyl-, sulfate- or glycuronyl groups in the small intestine and liver catalyzed by COMT, sulfotransferase (SULT) or UDP-glucuronosyltransfrease (UGT), respectively $(19,20,42)$. Considering some structural modification 
<smiles></smiles><smiles>COc1cc(-c2oc3cc(O)cc(OC)c3c(=O)c2O)ccc1O</smiles>
isorhamnetin<smiles></smiles>

4'-O-Methylquercetin or tamarixetin
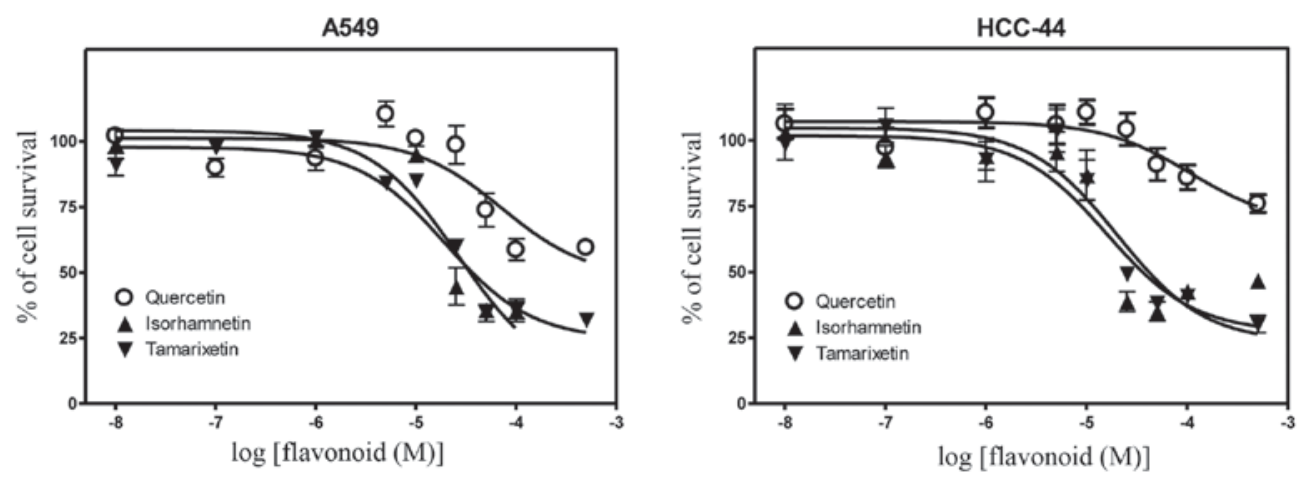

Figure 1. Dose-dependent inhibition curves of quercetin and its methylated metabolites in human lung adenocarcinoma cell lines A549 and HCC-44.
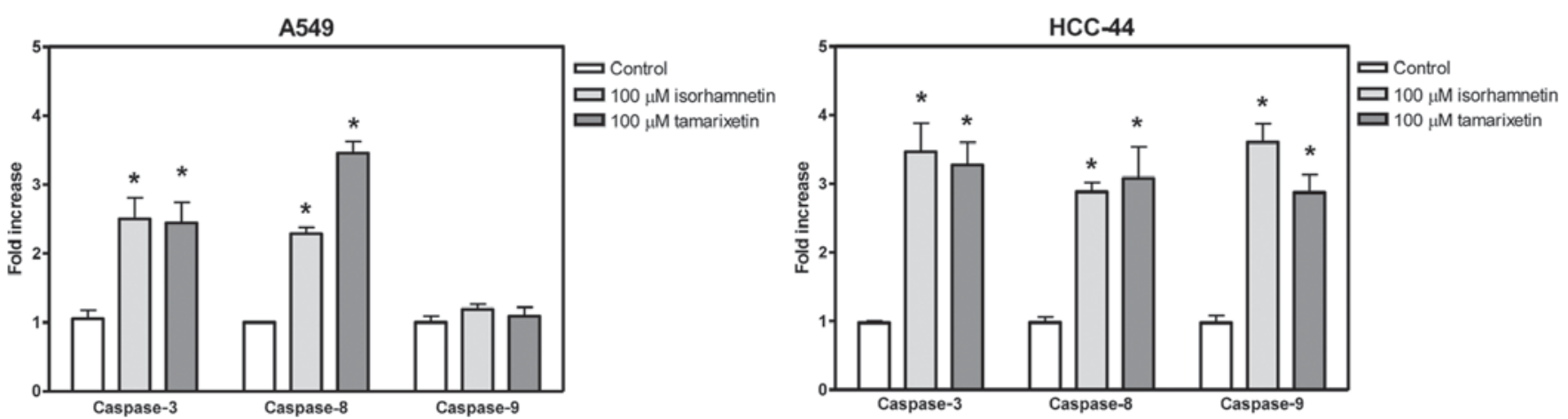

Figure 2. Activation of caspase family members after treatment of human lung adenocarcinoma cells A549 and HCC-44 with $100 \mu \mathrm{M}$ isorhamnetin and $100 \mu \mathrm{M}$ tamarixetin. ${ }^{*} \mathrm{P}<0.05$, methylquercetin-induced group vs. uninduced control group.

of initial compounds changes in their bioactivities could also be expected. Our results with two methyl conjugates of quercetin indeed confirm this standpoint indicating significantly higher antiproliferative potencies of both isorhamnetin and tamarixetin in HCC-44 and A549 lung adenocarcinoma cells compared to the parent flavonol. As the growth inhibitory effects of isorhamnetin have been previously reported in A549 cells (43-45), to the best knowledge of the authors this is the first study at all to describe the action of tamarixetin in human lung cancer cells. The results demonstrate that methylated metabolites of quercetin have considerably stronger anticancer activity than quercetin itself, whereas the potency does not depend on whether the methyl group is located in 3'-(isorhamnetin) or 4'-position (tamarixetin) of the B-ring in quercetin skeleton. In the future, it would be interesting to test the cytotoxic activity of other methylated derivatives of quercetin in lung cancer cell lines to study the possible structure-activity relationships. Furthermore, testing the potential antiproliferative action of other types of quercetin conjugates, i.e., sulfates and glucuronidates, would be equally important to better understand the anticarcinogenic role of flavonoids in the human body.

The two main mechanisms of cytotoxic action of flavonoids in malignant cells involve cell cycle arrest and induction of apoptosis. At that, apoptosis is largely mediated by two major routes: The intrinsic or mitochondrial signaling and extrinsic death receptor pathway. The former way is triggered by the release of cytochrome $c$ to the cytoplasm, cleavage of caspase- 9 and activation of caspase-3. The latter pathway includes the interaction with death receptor and sequential activation of caspase- 8 and caspase- 3 . In this study, we demonstrated that both methyl conjugates of quercetin, isorhamnetin and tamarixetin, induced apoptotic cell death in A549 and HCC-44 cells, characterized by the activation of effector caspase-3. However, as the cell death was predominantly mediated by extrinsic pathway in A549 cells, both extrinsic and intrinsic pathways were activated in HCC-44 cells by both methylated quercetin metabolites. The differences in activated caspase cascades in the tested cellular models can probably be caused by the different cellular signaling pathways triggered by quercetin 
derivatives. However, as A549 and HCC-44 lines were initially derived from a male and female lung adenocarcinoma patient, respectively, it is also possible that differences in the induced apoptotic pathways might involve some gender-specific aspects. Interestingly, the increase in cytotoxicity of methylquercetins compared to the parent quercetin molecule was significantly stronger (6.8-fold for isorhamnetin and 5.3-fold for tamarixetin) in female origin line HCC-44 than in male origin line A549 (2.7- and 3.7-fold increases, respectively). Therefore, it is possible that lung adenocarcinoma cells derived from men and women can behave differently to the treatment with methylated quercetins, a situation similar to the higher responses of female patients to the current therapeutic modalities in clinical use (7). These aspects clearly need further exploration. Moreover, considering that apoptosis has emerged as an important molecular mechanism for the anticancer action of standard chemotherapeutic drugs and novel candidate agents, further experiments for investigation of signaling pathways activated by methylated quercetins in different lung cancer cell lines are highly needed. In addition, the potential effects as well as possible toxicity issues of these compounds in xenograft rodent models also wait for testing.

Although the main aim of this work was to study the role of structural modification with adding a methyl group to quercetin molecule on its cytotoxic activity, this study has also several limitations. Among these, rate of apoptosis was not evaluated by flow cytometry and expression of pro- and anti-apoptotic proteins, such as Bax or Bcl-2, were not detected. Also, the level of apoptotic proteins, such as caspase- 3 and cleaved caspase-3, were not detected. Moreover, the effect of quercetin, isorhamnetin and tamarixetin on the cell cycle progression of A549 and HCC-44 lung adenocarcinoma cells as well as potential triggering of necrosis needs further unraveling in the future studies.

In conclusion, we showed that two methylated quercetin metabolites, isorhamnetin and tamarixetin, dose-dependently decreased the viability of A549 and HCC-44 lung adenocarcinoma cells at doses many-fold lower than those cytotoxically active for parent quercetin. Both metabolites also induced the apoptotic cell death in tested lung cancer experimental models revealing methylated quercetins as potential novel drug candidates for future treatment of non-small cell lung cancer.

\section{Acknowledgements}

This study was supported by the Estonian Society of Clinical Oncologists.

\section{References}

1. Lemjabbar-Alaoui H, Hassan OU, Yang YW and Buchanan P: Lung cancer: Biology and treatment options. Biochim Biophyc Acta 1856: 189-210, 2015.

2. Aggarwal A, Lewison G, Idir S, Peters M, Aldige C, Boerckel W, Boyle P, Trimble EL, Roe P, Sethi T, et al: The state of lung cancer research: A global analysis. J Thorac Oncol 11: 1040-1050, 2016.

3. McIntyre A and Ganti AK: Lung cancer-A global perspective. J Surg Oncol 115: 550-554, 2017.

4. Zappa C and Mousa SA: Non-small cell lung cancer: Current treatment and future advances. Transl Lung Cancer Res 5 : 288-300, 2016

5. Alberg AJ, Wallace K, Silvestri GA and Brock MV: Invited commentary: The etiology of lung cancer in men compared with women. Am J Epidemiol 177: 613-616, 2013.
6. Fu JB, Kau TY, Severson RK and Kalemkerian GP: Lung cancer in women: Analysis of the national surveillance, epidemiology and end results database. Chest 127: 768-777, 2005.

7. Cerfolio RJ, Bryant AS, Scott E, Sharma M, Robert F, Spencer SA and Garver RI: Women with pathologic stage I, II, and III non-small cell lung cancer have better survival than men. Chest 130: 1796-1802, 2006.

8. Schiller JH, Harrington D, Belani CP, Langer C, Sandler A, Krook J, Zhu J and Johnson DH; Eastern Cooperative Oncology Group: Comparison of four chemotherapy regimens for advanced non-small-cell lung cancer. N Engl J Med 346: 92-98, 2002.

9. Boffetta P, Couto E, Wichmann J, Ferrari P, Trichopoulos D, Bueno-de-Mesquita HB, van Duijnhoven FJ, Büchner FL, Key T, Boeing H, et al: Fruit and vegetable intake and overall cancer risk in the European Prospective Investigation into Cancer and Nutrition (EPIC). J Natl Cancer Inst 102: 529-537, 2010.

10. Miller AB, Altenhurg HP, Bueno-de-Mesquita B, Boshuizen HC, Agudo A, Berrino F, Gram IT, Janson L, Linseisen J, Overvad K, et al: Fruits and vegetables and lung cancer: Findings from the European Prospective Investigation into Cancer and Nutrition. Int J Cancer 108: 269-276, 2004.

11. Linseisen J, Rohrmann S, Miller AB, Bueno-de-Mesquita HB, Büchner FL, Vineis P, Agudo A, Gram IT, Janson L, Krogh V, et al: Fruit and vegetable consumption and lung cancer risk: Updated information from the European Prospective Investigation into Cancer and Nutrition (EPIC). Int J Cancer 121: 1103-1114, 2007.

12. Büchner FL, Bueno-de-Mesquita HB, Ros MM, Overvad K, Dahm CC, Hansen L, Tjønneland A, Clavel-Chapelon F, BoutronRuault MC, Touillaud M, et al: Variety in fruit and vegetable consumption and the risk of lung cancer in the European prospective investigation into cancer and nutrition. Cancer Epidemiol Biomarkers Prev 19: 2278-2286, 2010.

13. Wang M, Qin S, Zhang T, Song X and Zhang S: The effect of fruit and vegetable intake on the development of lung cancer: A meta-analysis of 32 publications and 20,414 cases. Eur J Clin Nutr 69: 1184-1192, 2015.

14. Zhou Y, Zheng J, Li Y, Xu DP, Li S, Chen YM and Li HB: Natural polyphenols for prevention and treatment of cancer. Nutrients 8: pii: E515, 2016.

15. Amararathna M, Johnston MR and Rupasinghe HP: Plant polyphenols as chemopreventive agents for lung cancer. Int J Mol Sci 17: pii: E1352, 2016.

16. Patil BS, Jayaprakasha GK, Chidambara Murthy KN and Vikram A: Bioactive compounds: Historical perspectives, opportunities, and challenges. J Agric Food Chem 57: 8142-8160, 2009.

17. Sak K: Cytotoxicity of dietary flavonoids on different human cancer types. Pharmacogn Rev 8: 122-146, 2014.

18. Cassidy A and Minihane AM: The role of metabolism (and the microbiome) in defining the clinical efficacy of dietary flavonoids. Am J Clin Nutr 105: 10-22, 2017.

19. Chen Z, Zheng S, Li L and Jiang H: Metabolism of flavonoids in human: A comprehensive review. Curr Drug Metab 15: 48-61, 2014.

20. Sak K: The Val158Met polymorphism in COMT gene and cancer risk: Role of endogenous and exogenous catechols. Drug Metab Rev 49: 56-83, 2017.

21. Cao Y, Chen ZJ, Jiang HD and Chen JZ: Computational studies of the regioselectivities of COMT-catalyzed meta-/para-O methylations of luteolin and quercetin. J Phys Chem B 118: 470-481, 2014.

22. Lieber M, Smith B, Szakal A, Nelson-Rees W and Todaro G: A continuous tumor-cell line from a human lung carcinoma with properties of type II alveolar epithelial cells. Int J Cancer 17: 62-70, 1976.

23. Gazdar AF, Girard L, Lockwood WW, Lam WL and Minna JD: Lung cancer cell lines as tools for biomedical discovery and research. J Natl Cancer Inst 102: 1310-1321, 2010.

24. Mosmann T: Rapid colorimetric assay for cellular growth and survival: Application to proliferation and cytotoxicity assays. J Immunol Methods 65: 55-63, 1983.

25. Kupcsik L: Estimation of cell number based on metabolic activity: The MTT reduction assay. Methods Mol Biol 740: 13-19, 2011.

26. Loizzo MR, Said A, Tundis R, Hawas UW, Rashed K, Menichini F, Frega NG and Menichini F: Antioxidant and antiproliferative activity of Diospyros lotus L. extract and isolated compounds. Plant Foods Hum Nutr 64: 264-270, 2009.

27. Tan J, Wang B and Zhu L: DNA binding, cytotoxicity, apoptotic inducing activity, and molecular modeling study of quercetin zinc(II) complex. Bioorg Med Chem 17: 614-620, 2009. 
28. Tan J, Wang B and Zhu L: DNA binding and oxidative DNA damage induced by a quercetin copper(II) complex: Potential mechanism of its antitumor properties. J Biol Inorg Chem 14: 727-739, 2009.

29. Tan J, Zhu L and Wang B: From GC-rich DNA binding to the repression of survivin gene for quercetin nickel(II) complex: Implications for cancer therapy. Biometals 23: 1075-1084, 2010.

30. Robaszkiewicz A, Balcerczyk A and Bartosz G: Antioxidative and prooxidative effects of quercetin on A549 cells. Cell Biol Int 31: 1245-1250, 2007.

31. Chan ST, Yang NC, Huang CS, Liao JW and Yeh SL: Quercetin enhances the antitumor activity of trichostatin A through upregulation of p53 protein expression in vitro and in vivo. PLoS One 8: e54255, 2013.

32. Katalinic M, Rusak G, Domaćinović Barović J, Sinko G, Jelić D, Antolović R and Kovarik Z: Structural aspects of flavonoids as inhibitors of human butyrylcholinesterase. Eur J Med Chem 45: 186-192, 2010.

33. Kawaii S, Tomono Y, Katase E, Ogawa $\mathrm{K}$ and Yano $\mathrm{M}$ Antiproliferative activity of flavonoids on several cancer cell lines. Biosci Biotechnol Biochem 63: 896-899, 1999.

34. Kim DH, Jung EA, Sohng IS, Han JA, Kim TH and Han MJ: Intestinal bacterial metabolism of flavonoids and its relation to some biological activities. Arch Pharm Res 21: 17-23, 1998

35. Hernandez J, Goycoolea FM, Quintero J, Acosta A, Castañeda M, Dominguez Z, Robles R, Vazquez-Moreno L, Velazquez EF, Astiazaran $\mathrm{H}$, et al: Sonoran propolis: Chemical composition and antiproliferative activity on cancer cell lines. Planta Med 73: $1469-1474,2007$

36. Said A, Tundis R, Hawas UW, El-Kousy SM, Rashed K, Menichini F, Bonesi M, Huefner A, Loizzo MR and Menichinib F: In vitro antioxidant and antiproliferative activities of flavonoids from Ailanthus excelsa (Roxb.) (Simaroubaceae) leaves. Z Naturforsch C 65: 180-186, 2010.
37. Han BJ, Li W, Jiang GB, Lai SH, Zhang C, Zeng CC and Liu YJ: Effects of daidzein in regards to cytotoxicity in vitro, apoptosis, reactive oxygen species level, cell cycle arrest and the expression of caspase and Bcl-2 family proteins. Oncol Rep 34: 1115-1120, 2015.

38. Yang Y, Zang A, Jia Y, Shang Y, Zhang Z, Ge K, Zhang J, Fan W and Wang B: Genistein inhibits A549 human lung cancer cell proliferation via miR-27a and MET signaling. Oncol Lett 12: 2189-2193, 2016.

39. Kano M, Takayanagi T, Harada K, Sawada S and Ishikawa F: Bioavailability of isoflavones after ingestion of soy beverages in healthy adults. J Nutr 136: 2291-2296, 2006.

40. Manach C, Williamson G, Morand C, Scalbert A and Rémésy C: Bioavailability and bioefficacy of polyphenols in humans. I. Review of 97 bioavailability studies. Am J Clin Nutr 81 (1Suppl): 230S-242S, 2005.

41. Lee J and Mitchell AE: Pharmacokinetics of quercetin absorption from apples and onions in healthy humans. J Agric Food Chem 60: 3874-3881, 2012.

42. Sak K and Everaus H: Sulfotransferase $1 \mathrm{~A} 1$ as a biomarker for susceptibility to carcinogenesis: From molecular genetics to the role of dietary flavonoids. Curr Drug Metab 17: 528-541, 2016.

43. Ruan Y, Hu K and Chen H: Autophagy inhibition enhances isorhamnetin-induced mitochondria-dependent apoptosis in non-small cell lung cancer cells. Mol Med Rep 12: 5796-5806, 2015.

44. Zhang BY, Wang YM, Gong G, Zhao H, Lv XY, Yuan GH and Han SR: Isorhamnetin flavonoid synergistically enhances the anticancer activity and apoptosis induction by cisplatin and carboplatin in non-small cell lung carcinoma (NSCLC). Int J Clin Exp Pathol 8: 25-37, 2015.

45. Li Q, Ren FQ, Yang CL, Zhou LM, Liu YY, Xiao J, Zhu L and Wang ZG: Anti-proliferation effects of isorhamnetin on lung cancer cells in vitro and in vivo. Asian Pac J Cancer Prev 16: 3035-3042, 2015. 